\title{
Características morfométricas, anatómicas y viabilidad de semillas de Cedrela odorata L. y Cariniana pyriformis Miers ${ }^{1}$
}

\section{Viability, morphometric, and anatomical characteristics of Cedrela odorata L. and Cariniana pyriformis Miers seeds}

\author{
Miguel Espitia-Camacho², Hermes Araméndiz-Tatis², Carlos Cardona-Ayala ${ }^{2}$
}

\begin{abstract}
Resumen
El objetivo del estudio fue describir las características morfométricas, anatómicas y la viabilidad de las semillas de Cedrela odorata y Cariniana pyriformis. El trabajo se llevó a cabo en la Universidad de Córdoba de Montería, Colombia, en el período comprendido entre abril de 2012 a julio de 2014; con una metodología descriptiva de laboratorio, la prueba de tetrazolio y la prueba de germinación en cámara germinativa. Se utilizaron semillas de lotes comerciales de tres localidades del departamento de Córdoba. Se seleccionaron al azar cinco árboles de cada especie y se tomaron al azar cinco muestras de cien semillas de cada uno. La descripción de la anatomía se hizo con base en diez semillas de cada especie. Para la prueba de tetrazolio, se estableció un experimento bajo un diseño completamente al azar, con seis tratamientos y cuatro repeticiones de veinticinco semillas. Los seis tratamientos se originaron al combinar las concentraciones de tetrazolio de $0,5,1,0$ y $1,5 \%$, con dos y tres horas de inmersión en la solución. Las características externas de $C$. odorata presentaron menor variación que las de $C$. pyriformis, especialmente aquellas relacionadas con el peso. Cuatro patrones topológicos fueron identificados en las semillas de cada especie. Se pudo determinar la viabilidad para $C$. pyriformis y $C$. odorata, con inmersiones por dos horas en concentraciones de $0,5 \mathrm{y}$ $1,0 \%$ de tetrazolio, respectivamente.
\end{abstract}

Palabras clave: calidad de semilla, germinación de semillas, prueba de tetrazolio.

\begin{abstract}
The aim of the study was to identify morphometric, anatomical, and the viability characteristics of the seeds of Cedrela odorata and Cariniana pyriformis. The study was carried out at the Universidad de Córdoba of Montería, Colombia, from April 2012 to July 2014; the study was carried out with a laboratory descriptive methodology, tetrazolium and germination tests in germination chamber were performed. Seeds of commercial lots from three locations in the department of Cordoba, were used. Five trees of each specie were randomly selected and from each tree, five samples of one hundred seeds, were randomly selected. The description of the shape and the anatomy of the seeds was made according to ten seeds of each specie. For the tetrazolium tests, a completely randomized design experiment, with six treatments, and four replications of 25 seeds, was established. The six treatments were originated
\end{abstract}

\footnotetext{
1 Recibido: 18 de setiembre, 2016. Aceptado: 16 de noviembre, 2016. Este trabajo formó partes de un proyecto de investigación financiado por la Universidad de Córdoba, Montería, Colombia.

2 Universidad de Córdoba. Carrera 6Nº76-103, Montería, Colombia. mespitia37@hotmail.com, haramendiz@hotmail.com, ccardonaayala@ yahoo.com
}

(C) 2017 Agronomía Mesoamericana es desarrollada en la Universidad de Costa Rica y se encuentra licenciada con Creative Commons Reconocimiento-NoComercial-SinObraDerivada 3.0 Costa Rica. Para más información escríbanos a pccmca@ucr.ac.cr 
by combining the tetrazolium concentrations of $0.5,1.0$, and $1.5 \%$, with two and three hours of immersion in the solution. The external characteristics of $C$. odorata showed less variation than $C$. pyriformis, especially in weight. Four topological patterns were identified in each specie. It was possible to determine the viability for $C$. pyriformis and $C$. odorata with immersion for two hours at concentrations of 0.5 and $1.0 \%$ tetrazolium, respectively.

Keywords: seed quality, seed germination, tetrazolium test.

\section{Introducción}

El cedro (Cedrela odorata L.) y el abarco (Cariniana pyriformis Miers) corresponden a dos especies forestales nativas del trópico americano, presentes en el departamento de Córdoba-Colombia, de las cuales se desconocen aspectos básicos relacionados con la morfología, germinación de las semillas y vigor de las plántulas. Dichos estudios son fundamentales para definir su multiplicación, establecimiento y regeneración natural, dado que existe una alta tasa de deforestación dentro de sus áreas de distribución (Gillies et al., 1997; Moreno y Del-Valle, 2015) y regeneración poco exitosa (Aguirre-Medina et al., 2014), por la extracción de árboles con características deseables.

Por las condiciones agroecológicas del departamento de Córdoba, estas especies han sido priorizadas para regeneración del bosque natural y establecimiento de plantaciones para producción de madera, por su adaptación, calidad de la madera y usos en muebles y acabados finos (Yaya et al., 2005; Luchi, 2011; Montiel et al., 2014). Sin embargo, el mayor esfuerzo de la investigación para fomentar el establecimiento de plantaciones forestales se ha dirigido principalmente hacia la selección de especies, la silvicultura, el desarrollo y la comercialización de los productos del bosque (Rodríguez, 2000), mientras que existen limitantes para el avance de sistemas de reproducción sexual y asexual, y el establecimiento de sistemas de certificación de calidad genética y física del material vegetal (Nieto, 2004).

Estudios relacionados con las características anatómicas, morfológicas, dimensiones y peso de las semillas de especies forestales nativas y exóticas, han sido realizados por varios autores, para la determinación de la viabilidad, germinación, comportamiento y adaptación de las especies en diferentes ecosistemas, conservación, uso en viveros y silvicultura (Oliveira et al., 2009). Entre las especies estudiadas se destacan Pinus brutia Ten. (Merve et al., 2015), Pinus tropicalis Mor. (Bonilla, 2014), Anadenanthera Colubrina (Vell.) Brenan (Barboza-Nogueira et al., 2014), Tabebuia roseo-alba (Ridl.) Sandwith (Abbade y Takaki, 2014), Stizolobium aterrimum Piper \& Tracy (Deminicis et al., 2014), Bumelia obtusifolia Roem et schult. var. excelsa (DC) Mig. (Nascimento, 2013), Plinia trunciflora (O. Berg) Kausel (Hössel et al., 2013), Gliricidia sepium (Jacq.) Steud. (Ribeiro-Reis et al., 2012), Jatropha curcas L. (Brenha et al., 2012), Copaifera langsdorffii Desf. \& Schizolobium parahyba (Vell.) S.F. Blake (Fogaça et al., 2011) y Ceiba speciosa (Lazarotto et al., 2011).

En Colombia, se destacan los trabajos realizados por Rodríguez (2000) en semillas de las especies Alnus jorullensis Humboldt, Bonpland \& Kunth, Cariniana piryformis Miers, Cedrela odorata L., Cordia alliodora (Ruiz \& Pav.) Oken, Tabebuia rosea (Bertol.) DC., Anacardium excelsum (Bertero\&Balb. ex Kunth) Skeels, Cedrela montana Moritz ex Turcz., Enterolobium cyclocarpum (Jacq.) Griseb., Erythrina edulis Triana ex M. Micheli, Erythrina fusca Lour., Jacaranda copaia (Aubl.) D. Don., Juglans neotropica Diels., Lafoensia speciosa (Kunth) DC., Samanea saman (Jacq.) Merrill y Tabebuia chrysantha (Jacq.) \& G. Nicholson.

La viabilidad de las semillas hace referencia a la capacidad de germinar y de originar plántulas normales en condiciones ambientales favorables; en consecuencia, la viabilidad de las accesiones se debe determinar antes de empacar y almacenar las semillas en el banco de germoplasma, y a intervalos regulares durante el almacenamiento (Rao et al., 2007). 
Existen varios métodos para determinar la viabilidad de las semillas, el más exacto y confiable es la prueba de germinación (Rao et al., 2007), el cual demanda plazos muy extensos que van desde los treinta días, dependiendo de la especie. La Asociación Internacional de Análisis de Semillas (ISTA, por sus siglas en inglés) solo acepta tres métodos rápidos de evaluación de la viabilidad como oficiales: la escisión del embrión, el ensayo topográfico de tetrazolio y el método de rayos x (ISTA, 2014). La prueba de tetrazolio se destaca por su velocidad, ya que los resultados se pueden obtener en aproximadamente veinticuatro horas, y su fiabilidad se ha probado en la evaluación de la calidad de semillas de especies forestales como Pinus pinea L. (Benito-Matías et al., 2004), Aspidosperma quebracho-blanco Schltr. (Alzugaray et al., 2006) y Tabebuia roseo-alba (Ridl.) Sandwith (Abbade y Takaki, 2014).

Debido a la importancia económica en el establecimiento de plantaciones comerciales para la producción de madera de calidad, y al valor ecológico en la regeneración del bosque natural de estas especies para el departamento de Córdoba, el objetivo del estudio fue describir las características morfométricas, anatómicas y la viabilidad de las semillas de C. odorata y C. pyriformis.

\section{Materiales y métodos}

\section{Localización}

La investigación se realizó entre abril de 2012 y julio de 2014, la caracterización morfométrica y los estudios de viabilidad de las semillas se realizaron en la Universidad de Córdoba de Montería, Colombia, ubicada en la zona media del valle del Sinú.

\section{Material genético}

Se utilizó semilla sexual de libre polinización de cinco árboles tomados al azar en cada uno de los lotes comerciales plantados con C. odorata en Montería (13 msnm; LN: 0842'27,73" y LO: 075'40'06,47') y en San Carlos (50 msnm; LN: 08²’23,67” y LO: 07540'13,19”); para C. pyriformis, la colecta se realizó sobre igual número de árboles en Tierralta (70 msnm; LN: 08 04'14,8" y LO: 076¹ $\left.11^{\prime} 30,1^{\prime \prime}\right)$, municipios ubicados en la zona ecológica bosque seco tropical con temperatura promedio de $27-28{ }^{\circ} \mathrm{C}$, humedad relativa de $84-86 \%$ y precipitación anual de 1200 - 1400 mm, en el departamento de Córdoba, Colombia (Palencia et al., 2006).

Dado que las dos especies son alógamas o prevalentemente alógamas (Trujillo, 2013), la semilla de cada árbol se consideró como una familia de medios hermanos. Al momento de la colecta de la semilla, los árboles presentaron edades entre 10 y 22 años, altura entre 8 y $18 \mathrm{~m}$, y diámetro a la altura del pecho (DAP) de 20 a $53 \mathrm{~cm}$.

\section{Caracterización morfométrica y anatómica de las semillas}

Para la caracterización morfométrica y anatómica de las semillas se utilizó una mezcla balanceada de 500 semillas de cada especie. Asimismo, se identificaron de forma precisa las partes esenciales de las semillas como embrión, endospermo y cubierta seminal, sobre una muestra aleatoria de diez semillas/especie, con base en la metodología propuesta por Martin (1946) y Niembro (1988) para semillas de árboles y arbustos.

Las características morfométricas relacionadas con ancho máximo (AS), largo máximo (LS), relación ancho/ largo (RALA), se midieron en cm; mientras que las asociadas con el peso de semilla (PES) y peso de cien semillas (P100S) se midieron en g. El número de semillas por kilogramo (NSKG) se estimó por conteo del número de semillas en cinco muestras de cien semillas/especie; luego el promedio se llevó a kg por el factor de expansión respectivo. 
Para la descripción anatómica y determinación de patrones topológicos en laboratorio se identificaron y describieron las partes esenciales anatómicas de diez semillas completas y sanas, tomadas al azar para cada especie. Para la descripción de la anatomía interna de forma muy nítida en cada especie se realizó un preacondicionamiento de las semillas, basado en remojo e inmersión por $12 \mathrm{~h}$ en agua destilada a temperatura entre $25^{\circ} \mathrm{C}$ y $30{ }^{\circ} \mathrm{C}$.

En las semillas de $C$. odorata se realizaron cortes longitudinales a través del embrión y se descartó la mitad de cada semilla. En las semillas de C.pyriformis, la separación de la cubierta seminal permitió una total exposición del embrión y de las partes consideradas "esenciales" y de importancia para la realización de la prueba de tetrazolio (ISTA, 2014). No se realizaron cortes longitudinales, debido a que, por tener un tipo de embrión axial-foliadoplegado, el corte causa una disgregación del mismo.

\section{Prueba de viabilidad}

La viabilidad de la semilla se determinó mediante la prueba topográfica de tetrazolio y la prueba de germinación. Para comprobar la viabilidad de las semillas, se realizó la tinción con tetrazolio, mediante inmersión de las mismas en solución al $1 \%$ por dos horas, en ausencia de luz y a una temperatura de $40{ }^{\circ} \mathrm{C}$ (ISTA, 2014). Luego se lavaron las semillas tres veces con agua destilada, para remover el exceso del colorante, y se evaluó la viabilidad con la ayuda de un estereoscopio para mejorar la visualización de las estructuras internas.

La clasificación de la viabilidad de las semillas, se realizó mediante la identificación de tres categorías recomendadas por Rao et al. (2007) para la interpretación de patrones de tinción:

Categoría 1. Semillas viables. Aquellas que presentaban: a) embrión y endospermo completamente teñidos; b) necrosis superficial en la mitad del endospermo, principalmente en las partes alejadas del embrión; c) áreas no teñidas (muertas) en el endospermo, en lugares opuestos a la radícula.

Categoría 2. Semillas no viables. Aquellas que presentaban: a) embrión y el endospermo sin teñir (muertas); b) embrión sin teñir aunque el endospermo estuviera teñido; c) necrosis aguda en el embrión; d) embrión teñido y endospermo sin teñir; e) necrosis en la punta de la radícula; f) daños graves en más de la mitad de las partes esenciales de la semilla.

Categoría 3. Semillas dudosas: semillas parcialmente teñidas producirán plántulas normales o anormales, dependiendo de la intensidad y patrón de la tinción; en esta categoría estuvieron las semillas que presentaron menos de la mitad teñida y con partes esenciales sanas.

Para evaluar la viabilidad de las semillas se estableció un experimento bajo un diseño completamente al azar, con seis tratamientos y cuatro repeticiones de veinticinco semillas cada una. Los tratamientos correspondieron a la combinación de tres concentraciones de tetrazolio $(0,5,1,0$ y $1,5 \%)$ y dos tiempos de tinción (dos y tres horas), estructurados como seis niveles de un factor. Para clasificar las semillas sobre la base de la intensidad y uniformidad del color (Pinto et al., 2009), se describieron los niveles de tinción que presentaron los embriones en cada tratamiento, los cuales fueron registrados fotográficamente con cámara digital. El porcentaje de semillas viables se obtuvo de las semillas de la categoría "viables", más la mitad de las semillas de la categoría "dudosas" (Rodríguez y Nieto, 1999).

Para medir la confiabilidad de la prueba de tetrazolio se evaluó la germinación de las semillas en condiciones de laboratorio. Se utilizó una cámara de germinación a una temperatura de $28{ }^{\circ} \mathrm{C}$, humedad relativa de $80 \%$, con periodo de luz de $10 \mathrm{~h} / \mathrm{d}$. Se utilizaron cuatro repeticiones de veinticinco semillas por cada especie, dispuestas sobre papel toalla en bandejas de aluminio; se le suministró riego uniforme durante 45 días. Se evaluó la viabilidad de las semillas con el registro del número de plántulas sanas emergidas durante el ensayo. 


\section{Análisis estadístico}

Para las características morfométricas se realizaron estadísticas descriptivas y se estimaron intervalos de confianza de 95\% de probabilidad. Para la estimación de los efectos de los tratamientos con tetrazolio, y de esta manera medir la confiabilidad de la prueba de tetrazolio en estas dos especies, se realizó un análisis de varianza de un factor con seis niveles (tratamientos) y la prueba de rango múltiple de Duncan al 5\% de probabilidad. Se utilizó el programa computacional GENES versión Windows (2004 .2 .1) desarrollado por Cruz (2004). La validación de la prueba de tetrazolio se hizo mediante la comparación múltiple de las medias de viabilidad de los seis tratamientos, más el promedio de viabilidad obtenido con la prueba convencional de germinación en cámara germinativa, como un tratamiento adicional.

\section{Resultados y discusión}

\section{Anatomía externa e interna de semillas}

La descripción morfométrica de las semillas de $C$. odorata identificaron una mayor variabilidad en los componentes derivados del peso de la semilla. Las características morfoanatómicas presentaron un ancho de semillas (AS) promedio de 0,90 $\pm 0,02 \mathrm{~cm}$, longitud de semillas (LS) promedio de 2,27 $\pm 0,01 \mathrm{~cm}$ incluyendo el ala, la relación de ambas características (RALA) mostró un valor promedio de 0,40 $\pm 0,02$; para peso de semilla (PES) la media estuvo en 0,01 $\pm 0,0008 \mathrm{~g}$ (Cuadro 1), muy diferente al reportado por Alderete-Chavez et al. (2005), para

Cuadro 1. Estadísticas descriptivas para las dimensiones y peso de las semillas de dos especies forestales (Cedrela odorata L. y Cariniana pyriformis Miers) nativas del departamento de Córdoba. Montería, Colombia. 2014.

Table 1. Descriptive statistics regarding seeds dimensions and weight of two native tree species (Cedrela odorata L. and Cariniana pyriformis Miers) found in Cordoba department. Montería, Colombia. 2014.

\begin{tabular}{|c|c|c|c|c|c|c|c|c|c|}
\hline \multirow{3}{*}{ Variable } & \multicolumn{9}{|c|}{ Cedrela odorata L. (Cedro) } \\
\hline & \multirow{2}{*}{$\begin{array}{c}\text { Datos } \\
(\#)\end{array}$} & \multirow[t]{2}{*}{ Media } & \multirow[t]{2}{*}{ Mín. } & \multirow[t]{2}{*}{ Máx. } & \multirow{2}{*}{$\begin{array}{l}\text { C.V. } \\
(\%)\end{array}$} & \multirow[t]{2}{*}{ Varianza } & \multirow{2}{*}{$\begin{array}{l}\text { Desviación } \\
\text { estándar }\end{array}$} & \multicolumn{2}{|c|}{ IC $(95 \%)$} \\
\hline & & & & & & & & LI & LS \\
\hline $\mathrm{AS}(\mathrm{cm})$ & 500 & 0,90 & 0,85 & 0,96 & 2,39 & 0,0005 & 0,02 & 0,899 & 0,910 \\
\hline LS (cm) & 500 & 2,27 & 2,19 & 2,71 & 4,40 & 0,0100 & 0,10 & 2,245 & 2,298 \\
\hline RALA & 500 & 0,40 & 0,33 & 0,50 & 5,22 & 0,0004 & 0,02 & 0,395 & 0,407 \\
\hline PES (g) & 500 & 0,01 & 0,0093 & 0,0136 & 7,49 & $6,4 \times 10^{-7}$ & 0,0008 & 0,011 & 0,011 \\
\hline P100S (g) & 500 & 1,10 & 0,93 & 1,35 & 7,49 & 0,0068 & 0,08 & 1,079 & 1,123 \\
\hline \multirow[t]{2}{*}{ NSKG (\#) } & 500 & 94965 & 79198 & 124091 & 8,54 & 65737963,3 & 8107,9 & 92724,7 & 97058,2 \\
\hline & \multicolumn{9}{|c|}{ Cariniana pyriformis Miers (Abarco) } \\
\hline $\mathrm{AS}(\mathbf{c m})$ & 500 & 0,69 & 0,58 & 0,80 & 6,03 & 0,0017 & 0,04 & 0,681 & 0,704 \\
\hline $\mathbf{L S}(\mathbf{c m})$ & 500 & 3,91 & 3,50 & 4,21 & 4,18 & 0,0266 & 0,16 & 3,860 & 3,947 \\
\hline RALA & 500 & 0,18 & 0,16 & 0,20 & 5,84 & 0,0001 & 0,011 & 0,180 & 0,186 \\
\hline PES (g) & 500 & 0,07 & 0,04 & 0,15 & 35,30 & 0,0006 & 0,024 & 0,062 & 0,075 \\
\hline P100S (g) & 500 & 6,91 & 4,26 & 15,07 & 35,32 & 5,9513 & 2,44 & 6,232 & 7,536 \\
\hline NSKG (\#) & 500 & 19498 & 7082 & 31390 & 29,60 & 33299230,0 & 5770,5 & 17903,5 & 20987,7 \\
\hline
\end{tabular}

AS = ancho de semilla; $\mathrm{LS}=$ longitud de semilla; RALA = relación ancho/largo de semilla; $\mathrm{PS}=$ peso de semillas; $\mathrm{P} 100 \mathrm{~S}=$ peso de cien semillas; NSKG= número de semillas por kilogramo; C.V.= coeficiente de variación; $\mathrm{IC}=$ intervalo de confianza; $\mathrm{LI}=$ límite inferior; LS= límite superior / AS = seed width; LS = seed length; RALA = seed width / length ratio; PS = seeds weight; P100S = weight of one hundred seeds; NSKG = number of seeds per kilogram; CV = coefficient of variation; $\mathrm{CI}=$ confidence interval; $\mathrm{LI}=$ lower limit; $\mathrm{LS}=$ upper limit. 
peso, longitud y ancho de las semillas, cuyos valores fueron $0,017 \mathrm{~g}, 2,60 \mathrm{~cm}$ y 3,64 mm, respectivamente; quienes señalaron la existencia de una gran variación genética en la especie. El peso promedio de cien semillas (P100S) fue de 1,10 $\pm 0,08 \mathrm{~g}$ y el número promedio de semillas/kg (NSKG) fue de $94965 \pm 8,108$, aproximadamente (Cuadro 1), lo cual está en función de su capacidad reproductiva, asociada a efectos genéticos y ambientales, debido a diferencias de viabilidad y fertilidad del polen, y receptividad estigmática (Mendizábal-Hernández et al., 2012; 2013).

En cuanto a la morfología ( $C$. odorata), las semillas fueron de forma ovoide, comprimidas, planas, provistas de un ala oscura, lisa y fácilmente quebradiza, la testa fue de color castaño (Figura 1a). En la estructura interna, el embrión fue axial, recto y espatulado, de color blanco o crema; con dos cotiledones grandes y planos. La radícula sobresalía y fue corta. El endospermo fue delgado, uniforme y blancuzco (Figura 1b), por lo que estas características concuerdan con las reportadas para esta misma especie (Stoffers, 1984; Montiel et al., 2014).

La descripción morfométrica de las semillas de $C$. pyriformis, presentaron dimensiones promedio para: AS de $0,69 \pm 0,04 \mathrm{~cm}$, LS incluyendo el ala de 3,91 $\pm 0,16 \mathrm{~cm}$, RALA de $0,18 \pm 0,011$, PES de 0,07 $\pm 0,024 \mathrm{~g}$, P100S de 6,91 $\pm 2,44 \mathrm{~g}$ (Cuadro 1). De acuerdo con el P100S, el NSKG fue de $19498 \pm 5771$ (Cuadro 1). Resultados similares han sido reportados en la misma especie por Rodríguez y Nieto (1999), Rodríguez (2000), Flores (2010) y Trujillo (2013), quienes encontraron variaciones significativas en los parámetros biométricos dentro de la semilla en una misma población.

Las semillas de $C$. pyriformis presentaron forma piramidal u obovada, con la extremidad micropilar aguda; la testa es dura y seca, de color café cuando estaba seca y se tornó negra cuando se humedeció; tenía un ala terminal (Figura 1c). La estructura interna mostró un embrión que, se puede clasificar de tipo axial subtipo plegado (Martin, 1946), estaba formado por el eje hipocótilo-radícula, con cotiledones de color crema y foliáceos, superpuestos, los cuales formaron pliegues bastante acentuados (Figura 1d). Estas características concuerdan con las reportadas por
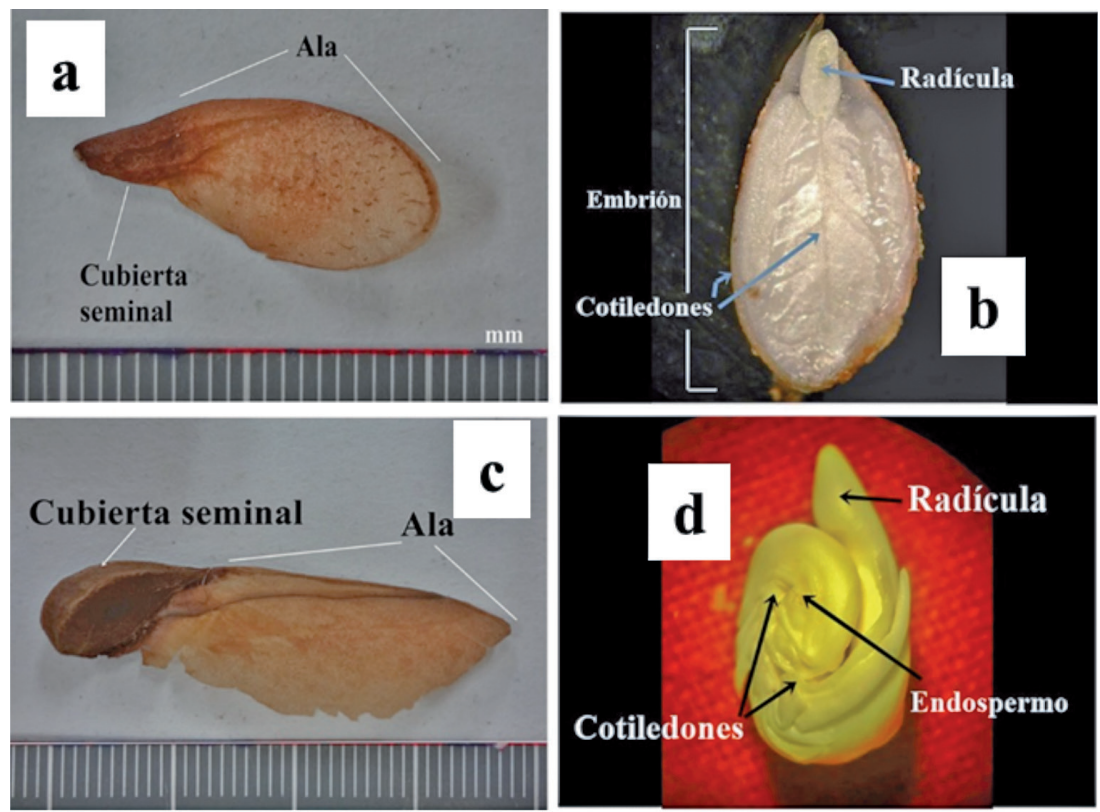

Figura 1. Anatomía externa e interna de las semillas de Cedrela odorata L. (a y b), y Cariniana pyriformis Miers (c y d). Montería, Colombia. 2014.

Figure 1. External and internal anatomy of the seeds of Cedrela odorata L. (a and b), and Cariniana pyriformis Miers (c and d). Montería, Colombia. 2014. 
Flores (2010) y Montiel et al. (2014), en investigaciones con semillas de Cariniana legalis y C. pyriformis, con algunas leves desviaciones.

\section{Determinación de patrones topológicos}

La descripción de los patrones de tinción identificados en las semillas de C. odorata y C. pyriformis están descritos y presentados gráficamente en las Figuras 2 y 3 . Estos fueron similares a los reportados en otras especies arbóreas como: T. roseoalba (Abbade y Takaki, 2014), Enterolobium contortisiliquum (Nogueira et al., 2014), Bumelia obtusifolia (Nascimento, 2013), Plinia trunciflora (Hössel et al., 2013) y Anadenanthera peregrina (Pinho et al., 2011).

\begin{tabular}{|c|c|c|c|c|}
\hline Clase & Viabilidad & Descripción & Fotografía & Esquema \\
\hline 1 & Viables & $\begin{array}{l}\text { Semillas con tinción total y } \\
\text { uniforme }\end{array}$ & & \\
\hline 2 & Viables & $\begin{array}{c}\text { Semillas con tinción en más del } \\
80 \% \text { de la radícula y el } \\
\text { embrión }\end{array}$ & & \\
\hline 3 & Dudosas & $\begin{array}{l}\text { Semillas teñidas en más del } \\
50 \% \text { de los cotiledones y } \\
\text { tinción rosada en el } 50 \% \text { de la } \\
\text { radícula }\end{array}$ & & \\
\hline 4 & Inviables & Semillas sin tinción & & \\
\hline
\end{tabular}

Figura 2. Patrones topológicos identificados en la prueba de tetrazolio en semillas de Cedrela odorata L. Montería, Colombia. 2014.

Figure 2. Topological patterns identified in the tetrazolium test performed on seeds of Cedrela odorata L. Montería, Colombia. 2014.

Las semillas registraron variaciones en la intensidad de la tinción, debido a que la sal de tetrazolio permite determinar la presencia, localización y naturaleza de las alteraciones de los tejidos de las semillas (Lima et al., 2010; ISTA, 2014), produciéndose en los tejidos vivos trifenil formazan, que identifica la actividad respiratoria de las mitocondrias, y como resultado muestra que hay viabilidad celular. Por lo tanto, el color rojo en los embriones es un indicador positivo de la viabilidad de las semillas (Craviotto et al., 2008), por lo que, se utiliza en programas de control de calidad de semillas; y aquellas regiones débilmente coloreadas en algunas partes del embrión, indican que las células presentan una disminuida actividad respiratoria y, por consiguiente, menor actividad de enzimas deshidrogenasas (Rao et al., 2007; Rodríguez et al., 2008). 


\begin{tabular}{|c|c|c|c|c|}
\hline Clase & Viabilidad & Descripción & Fotografía & Esquema \\
\hline $\mathbf{1}$ & Viables & $\begin{array}{c}\text { Semillas con tinción total y } \\
\text { uniforme }\end{array}$ & $\begin{array}{c}\text { Semillas con tinción en más del } \\
50 \% \text { de la radícula y con } \\
\text { cotiledones totalmente teñidos }\end{array}$ \\
\hline $\mathbf{2}$ & Viables & $\begin{array}{c}\text { Dudosas } \\
\text { Semillas con más del } 50 \% \text { de } \\
\text { lácula teñida y cotiledones } \\
\text { parcialmente teñidos }\end{array}$ & \\
\hline $\mathbf{4}$ & Inviables & Semillas sin tinción & \\
\hline
\end{tabular}

Figura 3. Patrones topológicos identificados en prueba de tetrazolio en semillas de Cariniana pyriformis Miers. Montería, Colombia. 2014.

Figure 3. Topological patterns identified in the tetrazolium test performed on seeds of Cariniana pyriformis Miers. Montería, Colombia. 2014.

Evaluación de diferentes concentraciones de tetrazolio y tiempos de tinción para medir la viabilidad de las semillas

El análisis de varianza de cada especie en los patrones topológicos y los resultados de las pruebas de separación de medias están consignados en el Cuadro 2. En las semillas de C. pyriformis los tratamientos no presentaron diferencias significativas en ninguna de las categorías estimadas, lo cual evidencia que las concentraciones de tetrazolio evaluadas y los tiempos de tinción surtieron el mismo efecto en términos de coloración de los tejidos esenciales de la semilla. Sin embargo, al examinar la calidad de la tinción por tratamiento (Figura 4), se pudo apreciar que las diferencias cualitativas no fueron muy marcadas, y todas las combinaciones permitieron una observación clara y confiable de la tinción en los tejidos vivos de la semilla, de acuerdo con lo recomendado por Moore (1985), Rodríguez y Nieto (1999) e ISTA (2014).

Estos resultados permitieron inferir que, cualquiera de estos tratamientos fue eficiente y confiable para determinar la viabilidad de las semillas de C. pyriformis, lo que es concordante con lo reportado por Grzybowski et al. (2012) en Hordeum vulgare; por lo tanto, utilizar la solución de tetrazolio a concentraciones de 0,5\% por dos horas, es más económico y permite una tinción adecuada de los tejidos de las semillas, sin perjudicar la visualización de la viabilidad. Resultados semejantes han sido sugeridos en las especies Acca sellowiana O. Berg Burret (Sarmento et al., 2013), Ceiba speciosa (Lazarotto et al., 2011), Copaifera langsdorffii, Schizolobium 
Cuadro 2. Valores medios de germinación y de viabilidad con diferentes concentraciones de tetrazolio en dos especies forestales Cedrela odorata L. y Cariniana pyriformis Miers, nativas del departamento de Córdoba. Montería, Colombia. 2014.

Table 2. Average germination values and viability according to different concentrations of tetrazolium for two forest species: Cedrela odorata L. and Cariniana pyriformis Miers, natives species of Cordoba department. Monteria, Colombia. 2014.

\begin{tabular}{lcc}
\hline TRAT & \multicolumn{2}{c}{ Viabilidad (\%) } \\
\cline { 2 - 3 } & Cariniana pyriformis & Cedrela odorata \\
\hline PG & $89 \mathrm{a}$ & $82 \mathrm{~b}$ \\
{$[0,5 \%]-2 \mathrm{~h}$} & $94 \mathrm{a}$ & $90 \mathrm{a}$ \\
{$[0,5 \%]-3 \mathrm{~h}$} & $92 \mathrm{a}$ & $94 \mathrm{a}$ \\
{$[1,0 \%]-2 \mathrm{~h}$} & $93 \mathrm{a}$ & $86 \mathrm{~b}$ \\
{$[1,0 \%]-3 \mathrm{~h}$} & $85 \mathrm{a}$ & $71 \mathrm{c}$ \\
{$[1,5 \%]-2 \mathrm{~h}$} & $92 \mathrm{a}$ & $82 \mathrm{~b}$ \\
{$[1,5 \%]-3 \mathrm{~h}$} & $95 \mathrm{a}$ & $84 \mathrm{~b}$ \\
$\mathrm{Pr}>\mathrm{F}$ & $\mathrm{ns}$ & $*$ \\
$\mathrm{CV}(\%)$ & 10,7 & 8,3 \\
\hline
\end{tabular}

$\mathrm{PG}=$ porcentaje de germinación en cámara germinativa; C.V. = coeficiente de variación $/$; PG = germination percentage found in germinative chamber; C.V. = coefficient of variation.

Medias con la misma letra no difieren significativamente al 5\% de probabilidad, según la prueba de Duncan / Means with the same letter do not significantly differ at $5 \%$ probability, according to Duncan's test.
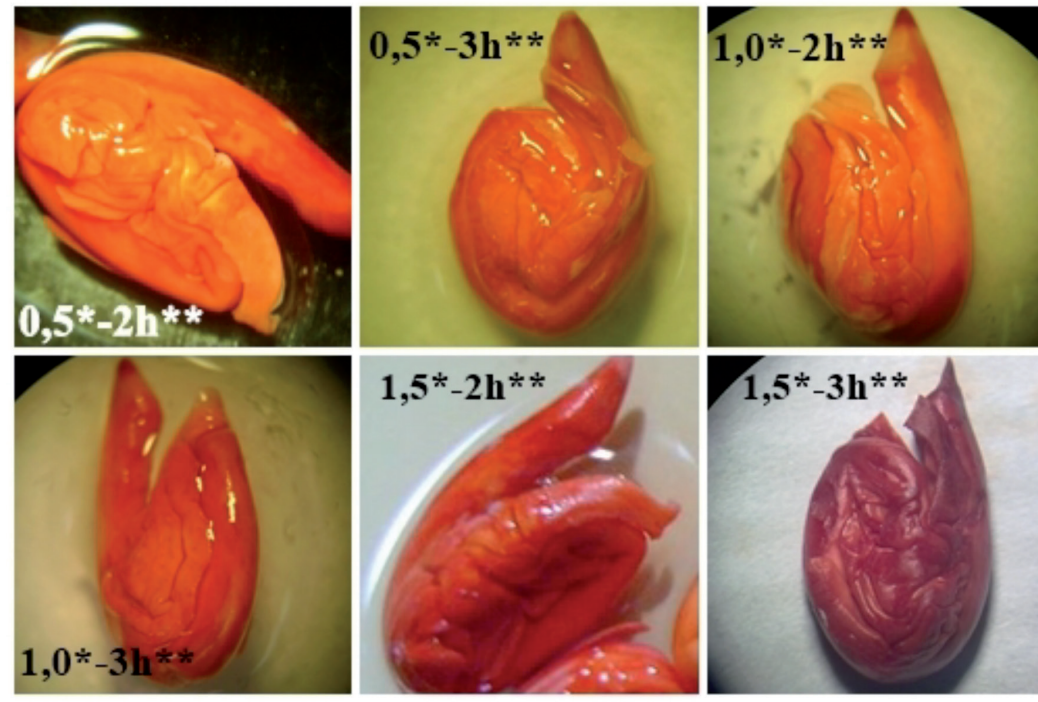

Figura 4. Semillas de Cariniana pyriformis Miers, sometidas a la prueba de tetrazolio. Departamento de Córdoba, Montería, Colombia. 2014. *concentración de tetrazolio en \%; **tiempo de tinción en horas.

Figure 4. Seeds of Cariniana pyriformis Miers, that experienced the tetrazolium test. Córdoba Department, Montería, Colombia. 2014.

* Tetrazolium concentration in \%; ** Staining time in hours. 
parahyba (Fogaça et al., 2011), Annona cherimola Mill. x Annona squamosa L. (Gimenez et al., 2014), Stizolobium aterrimum (Deminicis et al., 2014) y Crambe marítima L. (Guimarães et al., 2015).

Lo antes anotado, se ratificó con los resultados obtenidos en la prueba de germinación en cámara germinativa, cuyo porcentaje de plántulas germinadas $(85 \%-95 \%)$, no presentó diferencias significativas con los porcentajes de semillas viables estimados mediante los diferentes tratamientos de la prueba bioquímica de tetrazolio (Cuadro 2). La presencia de semillas inviables, obedeció a la degradación de las membranas celulares por peroxidación de lípidos y peroxidación no enzimática, que son factores que contribuyen a la degradación de la viabilidad de las semillas (Ravikumar et al., 2002).

El análisis de varianza para $C$. odorata, registró diferencias significativas y altamente significativas para semillas viables e inviables, respectivamente, más no así para las semillas de la categoría dudosas (Cuadro 2). La comparación de medias (Cuadro 2) y las intensidades de tinción de los embriones (Figura 5) resaltaron que la concentración de $1 \%$ de tetrazolio y tiempo de tinción de dos horas, fueron eficientes para evaluar la calidad de semillas, dado que las primeras horas de absorción de agua por la semilla son importantes, ya que se relacionan con la actividad enzimática y por lo tanto, con la coloración final (Lima et al., 2010). Las concentraciones de 0,5\% por dos y tres horas fueron las que presentaron los mayores porcentajes de viabilidad; sin embargo, están sobre estimadas (al comparar con la capacidad germinativa), mientras que al 1\% por tres horas subestima el porcentaje de viabilidad. La concentración de tetrazolio al 1\% durante dos horas, se ratificó mediante las pruebas de germinación, en las cuales los porcentajes estimados no presentaron diferencias significativas con los valores obtenidos a través de los tratamientos de la prueba bioquímica (Cuadro 2), y fueron consistentes con los reportados en ahuyama (Barros et al., 2005) y en pepino (Lima et al., 2010).

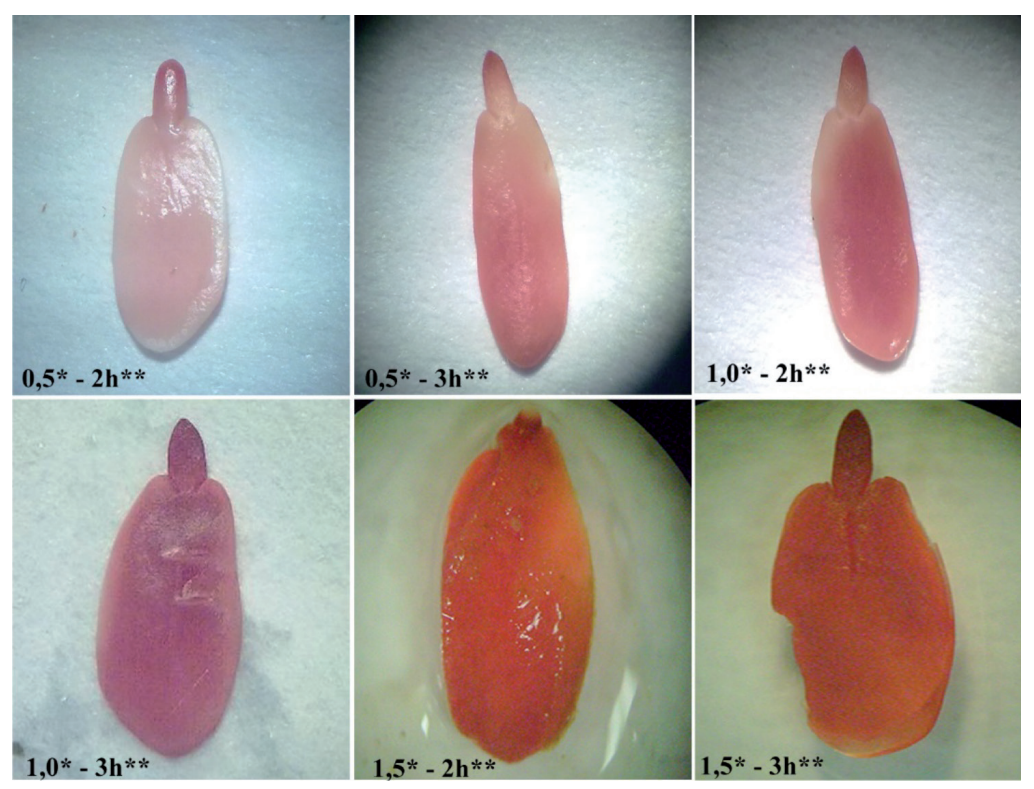

Figura 5. Semillas de Cedrela odorata L. sometidas a la prueba de tetrazolio. Departamento de Córdoba, Montería, Colombia. 2014. *concentración de tetrazolio en \%; **tiempo de tinción en horas.

Figure 5. Cedrela odorata L. seeds under the tetrazolium test. Córdoba Department, Montería, Colombia. 2014.

* Tetrazolium concentration in \%;* Staining time in hours. 


\section{Literatura citada}

Abbade, L.C., e M. Takaki. 2014. Teste de tetrazólio para avaliação da qualidade de sementes de Tabebuia roseo-alba (Ridl.) Sandwith - Bignoniaceae, submetidas ao armazenamento. Rev. Árvore 38:233-240.

Aguirre-Medina, J.E., F. Mina-Briones, J. Cadena-Iñiguez, J.D. Dardón-Zunun, y D. Hernández-Sedas. 2014. Crecimiento de Cedrela odorata L. biofertilizada con Rhizophagus intraradices y Azospirillum brasilense en vivero. Rev. Chapingo Ser. Cienc. Fores. Amb. 20:177-186.

Alderete-Chávez, A., N. Landero, y J.E. González de la Torre. 2005. Variación en semillas de Cedrela odorata L. procedentes de los estados de Campeche y Tabasco, México. Foresta Veracruzana 7(2):41-44.

Alzugaray, C., N.J. Carnevale, A.R. Salinas, y R. Pioli. 2006. Calidad de semillas de Aspidosperma quebracho-blanco Schltr. Quebracho 13:26-35.

Barboza-Nogueira, F.C., C.H. Lobo-Pinheiro, S. Medeiros-Filho, and D.M. Da-Silva-Matos. 2014. Seed germination and seedling development of Anadenanthera Colubrina in response to weight and temperature conditions. J. Plant Sci. 2:3742.

Barros, D.I., D.C.F.S. Dias, e M.C. Bhering. 2005. Uso do teste de tetrazólio para avaliação da qualidade de sementes de abobrinha. Rev. Bras. Sementes 27(2):165-171.

Benito-Matías, L.F., N. Herrero-Sierra, I. Jiménez, y J.L. Peñuelas-Rubira. 2004. Aplicación de métodos colorimétricos para la determinación de la viabilidad en semillas de Pinus pinea: test de tetrazolio e índigo carmín. Cuad. Soc. Esp. Cien. For. $17: 23-28$.

Bonilla, V.M. 2014. Variación del peso y viabilidad de las semillas de Pinus tropicalis para diferentes procedencias. Rev. Cub. Cienc. For. 2:1-10.

Brenha, J., N. De-Oliveira, A. Cândido, A. Godoy, e C. Alves. 2012. Teste de tetrazólio em sementes de pinhão manso. Visão Acadêmica 13(4):63-79.

Craviotto, M., M. Arango, and C. Gallo. 2008. Topographic tetrazolium test for soybean. Ediciones INTA, ARG. http://www. cosechaypostcosecha.org/data/articulos/calidad/TopographicTetrazoliumTestForSoybean.asp (consultado 8 abr. 2011)

Cruz, C. 2004. Programa genes. Versão Windows. Aplicativo computacional em genética e estatística. Universidade Federal de Viçosa, BRA. http:///www.ufv.br/dbg/genes/genes.htm (consultadas 5 jul. 2007).

Deminicis, B.B., P.D.R. Rodrigues, B.P. Faria, H.D. Vieira, H.D. PandolfI-Filho, and G.S. Freitas. 2014. Tetrazolium test to evaluate Stizolobium aterrimum seeds quality. Am. Plant Sci. 5:148-152.

Flores, E. 2010. Biología de las semillas. En: J.A. Vozzo, editores, Manual de semillas de árboles tropicales. USDA, MO, USA. p. $9-120$.

Fogaça, C.A., N.G Krohn, M.A. Souza, e R.C. Paula. 2011. Teste de tetrazólio em sementes de Copaifera langsdorffii e Schizolobium parahyba. Floresta, Curitiba 41:895-904.

Gillies, A.C.M., J.P. Cornelius, A.C. Newton, C. Navarro, M. Hernández, and J. Wilson. 1997. Genetic variation in Costa Rican population of the tropical timber species Cedrela odorata L. assessed using RAPDS. Mol. Ecol. 6:1133-1145.

Gimenez, J., G. Ferreira, e C. Cavariani. 2014. Teste de tetrazólio para a avaliação da viabilidade de sementes de atemoia (Annona cherimola Mill. $\times$ A. squamosa L.). J. Seed Sci. 36:357-361.

Guimarães, R.R., L. Leão, M.C. Nery, A.R. De Souza, S.M. Cruz, e P.C.A. De Resende. 2015. Tetrazolium test in crambe seeds. Semin: Ciênc. Agrár. 36:2539-2544. doi:10.5433/1679-0359.2015v36n4p2539 
Grzybowski, C., J.S.A Alves-de-Oliviera, R.S. Silva, and M. Panobianco. 2012. Viability of barley seeds by the tetrazolium test. Rev. Bras. Sementes 34:47-54.

Hössel, C.J., J.S.A. Alves-de-Oliveira, K.C. Fabiane, A. Wagner-Júnior, e I. Citadin. 2013. Conservação e teste de tetrazólio em sementes de jabuticabeira. Rev. Bras. Frutic. 35:255-261. doi:10.1590/S0100-29452013000100029

ISTA (International Seed Testing Association). 2014. International rules for seed testing. 2014 ed. ISTA, Bassersdorf, SUI.

Lazarotto, M., G. Piveta, M. Muniz, e L. Reiniger. 2011. Adequação do teste de tetrazólio para avaliação da qualidade de sementes de Ceiba speciosa. Semina: Ciênc. Agrár. 32:1243-1250.

Lima, L.B., T.L. Pinto, e A.D. Novembre. 2010. Avaliação da viabilidade e do vigor de sementes de pepino pelo teste de tetrazolio. Rev. Bras. Sementes 32:60-68.

Luchi, A.E. 2011. Quantitative features of Cedrela odorata L. wood (Meliaceae). Rev. Bras. Bot. 34:403-410.

Martin, A. 1946. The comparative internal morphology of seeds. Am. Midl. Nat. 36:513-660.

Mendizábal-Hernández, L. Del C., J.J. Hernández-Viveros, y J. Alba-Landa. 2013. Estudio de conos y semillas de Cedrela odorata L. en una generación parental y una generación filial. Foresta Veracruzana 15(1):45-51.

Mendizábal-Hernández, L. Del C., J.J. Hernández-Viveros, J. Alba-Landa, E. Ramírez-García, y H. Cruz-Jiménez. 2012. Potencial y eficiencia de producción de semillas de Cedrela odorata L. Foresta Veracruzana 14(2):31-36.

Merve, D.M., N. Seyedi, and N. Bilir. 2015. Seedling quality and morphology in seed sources and seedling type of brutian pine (Pinus brutia Ten.). World J. Agric. Res. 3(2):83-85.

Montiel, J., M. Espitia, y H. Araméndiz. 2014. Secado, tolerancia a la desecación y viabilidad de semillas de cinco especies forestales nativas en Córdoba. En: R. Cabrales-Rodríguez, editor, Memorias XLIV Congreso Nacional de COMALFI (Sociedad Colombiana de Control de Malezas y Fisiología Vegetal): la fisiología vegetal en la producción de las plantas forrajeras. COMALFI, Montería, COL. p. 48.

Moore, R.P. 1985. Handbook on tetrazolium testing. ISTA, Zürich, SUI.

Moreno, M.M., and J.I. Del-Valle. 2015. Influence of local climate and ENSO of the growth of abarco (Cariniana pyriformis) in Choco, Colombia. Trees 29:97-107.

Nascimento, I. 2013. Determinação de metodologias para teste de germinação e vigor de sementes de quixabeira (Bumelia obtusifolia Roem et schult. var. excelsa (DC) Mig.). Rev. Árvore 37:701-706.

Niembro, A. 1988. Semillas de árboles y arbustos: Ontogenia y estructura. Editorial Limusa, MEX.

Nieto, V. 2004. Las diez especies top para investigación y desarrollo forestal. Rev. M\&M 43:11-17.

Nogueira, N.W., S.T. Barros, e R.M. Oliveira. 2014. Tetrazolium test in timbaúba seeds. Semin: Ciênc. Agrár. 35:2967-2976.

Oliveira, C.M., C.C. Martins, e J. Nakagawa. 2009. Método de preparo das sementes de mamoneira (Ricinus communis L.) para o teste de tetrazólio. Rev. Bras. Sementes 31:160-167.

Palencia, G., T. Mercado, y E. Combatt. 2006. Estudio agroclimático del departamento de Córdoba. Universidad de Córdoba, COL.

Pinho, D.S., E.E. De Lima, A.P. Vilela, e V.C. Borges. 2011. Adequação da metodología do teste de tetrazólio para avaliação da viabilidade de sementes de angico. Pesq. Flor. Bras. 67:269-272.

Pinto, T.L.F., J.M. Filho, V.A. Forti, C. De Carvalho, e F.G. Gomes. 2009. Avaliação da viabilidade de sementes de pinhão manso pelos testes de tetrazólio e de raios X. Rev. Bras. Sementes 31:195-201.

Rao, N.K., J. Hanson, M.E. Dulloo, K. Ghosh, D. Novell, y M. Larinde. 2007. Manual para el manejo de semillas en bancos de germoplasma. No. 8. Bioversity International, Roma, ITA. 
Ravikumar, R., G. Ananthakrishnan, S. Girija, and A. Ganapathi. 2002. Seed viability and biochemical changes associated with accelerated ageing in Dendrocalamus strictus seeds. Biol. Plant. 45:153-156. doi:10.1023/A:1015106203273

Ribeiro-Reis, R., C. Pelacani, C. Antunes, B. Dantas, and R. De-Castro. 2012. Physiological quality of Gliricidia sepium (Jacq.) Steud. (Leguminosae - Papilionoideae) seeds subjected to different storage conditions. Rev. Árvore 36:229-235.

Rodríguez, J. 2000. Protocolos de germinación para la certificación de semillas forestales. Serie técnica N46. CONIF, Bogotá, COL.

Rodríguez, I., G. Adam, y J.M. Durán. 2008. Ensayos de germinación y análisis de viabilidad y vigor en semillas. Agricultura 912:836-842.

Rodríguez, J., y V. Nieto. 1999. Investigación en semillas forestales nativas. CONIF, Bogotá, COL.

Sarmento, M.B., A.C. Silva, F. Amaral-Villela, K. Santos, e L. Pérez. 2013. Teste de tetrazólio para avaliação da qualidade fisiológica em sementes de goiabeira-serrana (Acca sellowiana O. Berg Burret). Rev. Bras. Frutic. 35: 270-276.

Stoffers, A.L. 1984. Cederela odorata L. En: J.A. Vozzo, editor, Manual de semillas de árboles tropicales. USDA, MO, USA. p. $375-378$.

Trujillo, E. 2013. Guía de reforestación. $3^{\text {a }}$ ed. DAYBERMEDIOS. SAS, Bogotá, COL.

Yaya, M.L., O.L. Rodríguez, W. Usaquén, y A. Chaparro. 2005. Inducción de organogénesis indirecta en Abarco (Cariniana pyriformis Miers.). Agron. Colomb. 23:50-54. 